\title{
NOTIFIABLE DISEASES DATABASE SYSTEM: REVIEW AND DEVELOPMENT STRATEGY
}

Lina Persson and Mark Bartlett Communicable Diseases Branch NSW Department of Health

\section{BACKGROUND}

The Communicable Diseases Branch of the NSW Department of Health recently undertook a review of the existing notification system, to determine its effectiveness and guide future development of the system. This report briefly outlines the nature of the review, its recommendations, and the progress to date in addressing the recommendations. A more complete description of the nature and outcome of this process will be presented in a future edition of the NSW Public Health Bulletin.

\section{THE CURRENT SYSTEM}

Under the NSW Public Health Act 1991, certain Scheduled Medical Conditions (SMCs) are required to be notified. The Notifiable Diseases Database (NDD) system is a tool for maintaining data related to notifiable communicable conditions. The primary objective of the NDD system is to provide timely and accurate data on notifiable communicable diseases in a flexible and secure manner.

The NDD system has been operational for over 10 years in a number of forms. The current system has been operational since 1997, with several updates.

The system covers all 17 area health services in NSW, and since early 2003 has also included Corrections Health as a separate area health service. Information on over 40 notifiable SMCs is maintained by the system, with over 30,000 cases notified in 2003. On average, 600 new records are added to the system every week.

To guide public health action, the NDD system encompasses the collection, analysis, interpretation, and dissemination of data regarding certain SMCs. The information generated by this system is used to:

- record the public health interventions and outcomes associated with the management of each case of a notified condition;

- assess the current status of individual conditions or disease groups;

- identify specific groups at risk;

- provide quantitative evidence related to control strategies;

- understand the epidemiology of specific conditions;

- inform the development of prevention strategies and policies.

- prioritise the allocation of resources.

\section{STRUCTURE OFTHE NOTIFIABLE DISEASES DATABASE SYSTEM}

The goal of any information system is the manufacture of appropriate information products for end users. Data must be captured and prepared for processing by the input activity. Data are typically subjected to transforming activities such as calculating, comparing, sorting, classifying, and summarising. Information in various forms is transmitted to users and made available in the output activity. Data storage ensures that information is retained in an organised manner for later use. An information system should produce feedback regarding its input, processing, output, and storage activities in order to control performance. ${ }^{1}$

The NDD system can therefore be considered as having the following components:

- system control;

- data input;

- data transformation;

- information output;

- system architecture.

\section{REVIEW METHODOLOGY AND FINDINGS}

In October 2002 the Communicable Diseases Branch of the NSW Department of Health engaged the services of a consultant to evaluate the effectiveness of the NDD and make recommendations on the future development of the system.

All information systems use people, hardware, software, network and knowledge resources to perform input, transformation, output, storage and control activities that convert data resources into information products. ${ }^{1}$ Multiple sources of information from all these interrelated elements were needed to construct a balanced and reliable description of the system and provide a frame of reference for evaluating the system.

Representatives of stakeholder groups were identified to provide input into the review. Stakeholders included:

- system control and system architecture-staff from the Communicable Diseases Branch and the Enterprise Information Technology Branch;

- data input-public health unit surveillance staff and directors, pathology laboratories, medical practitioners, schools, and hospitals.

- data processing and information output-staff from the Commonwealth Department of Health and Ageing, the Centre for Epidemiology and Research, the AIDS and Infectious Diseases Branch, advisory committees, and the public. 


\section{FIGURE 1}

THE RELATIONSHIP BETWEEN STAKEHOLDERS INVOLVED WITHTHE NOTIFIABLE DISEASES DATABASE SYSTEM AND THE GENERIC FLOW OF DATA WITHIN THE SYSTEM

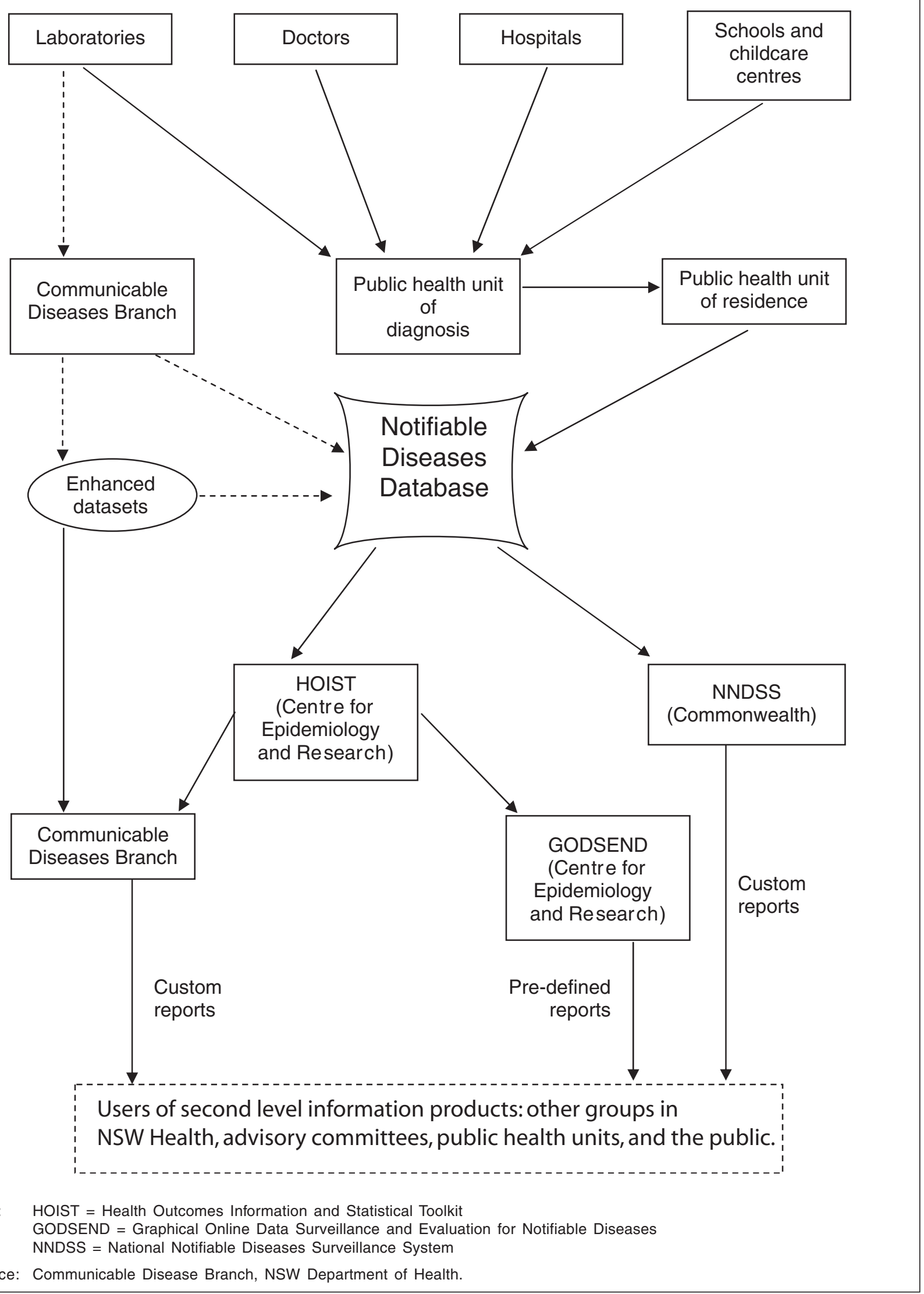


An evaluation form was developed and stakeholder representatives were invited to use this to provide their perspective on the NDD system. A number of key stakeholders were also interviewed to collect information on operational aspects of the system. Existing documentation and current work practices were also reviewed.

\section{ANALYSIS}

The information collected from these sources was used to assess the effectiveness of the system by comparing current operations with established objectives and specific stakeholder requirements. In accordance with the Guidelines for Evaluating Public Health Surveillance Systems from the United States Centre for Disease Control and Prevention, ${ }^{2}$ the following performance characteristics were considered: simplicity, flexibility, data quality, acceptability, sensitivity, predictive value positive (PVP), representativeness, timeliness and stability.

\section{FINDINGS}

The review found that the NDD system is currently limited in its ability to meet its primary objective of providing timely and accurate data on notifiable conditions in a flexible and secure environment.

The following barriers to further development were identified:

- the roles and responsibilities of different stakeholder groups involved with the system are unclear;

- the nature and type of data collected in the system has grown, and will continue to grow, in an informal way. The existing system cannot readily facilitate change, so alterations are resource intensive and untimely;

- data do not flow through the system consistently and is subject to different transformation rules depending on the way information is accessed.

The recommendations from the review were categorised into those relating to improving the existing system, and those guiding the development of a new system.
Recommendations to improve the existing database cover all aspects of the system from system control, data input, data transformation, and information output through to system architecture. The proposed improvements to the existing system will also clarify the requirements of a new system.

Recommendations guiding the development of a new system included the preliminary phase of a comprehensive systems analysis, which should encompass the principles of problem definition, system specification, system design, system development, and ongoing review and maintenance.

\section{PROGRESS ON IMPLEMENTING THE REVIEW RECOMMENDATIONS}

To date, several of the review recommendations have been implemented or are in the process of being implemented. These include:

- rationalising and strengthening the documentation surrounding the existing system;

- reviewing the existing network infrastructure in relation to capacity to support a web based information system;

- evaluating the feasibility of electronic transfer of laboratory-based notifications;

- reviewing privacy and security requirements of a notifiable conditions system;

- developing data conventions to ensure consistent data flow and processing for all notifiable conditions.

In the coming months, projects addressing these recommendations will be finalised and the remaining recommendations will be implemented.

\section{REFERENCES}

1. O'Brien J. Management of Information Systems. McGrawHill and Irwin, 2002.

2. CDC Guidelines Working Group. Updated Guidelines for Evaluating Public Health Surveillance Systems. Atlanta: United States Centers for Disease Control and Prevention, July 2001. 